\title{
COMMENT
}

\section{Improving VLBW infant outcomes with big data analytics}

\author{
F. Sessions Cole $^{1}$ \\ Pediatric Research (2021) 90:20-21; https://doi.org/10.1038/s41390-021-01507-5
}

Big data analytic strategies applied to routinely acquired, continuously assessed physiologic monitoring parameters in the neonatal intensive care unit (NICU) have provided clinically useful, predictive algorithms that reduce neonatal morbidities through detection of vital sign patterns associated with adverse outcomes. ${ }^{1-6}$ For example, based on large clinical trials carried out in low birth weight infants, ${ }^{7-10} \mathrm{NICU}$ guidelines target gestational and chronologic age-specific oxygen saturation limits that reduce risk of neurologic morbidities, retinopathy of prematurity, oxygen radical injury, necrotizing enterocolitis, and death. 5,6,11 These studies highlight the usefulness of standardized, predictive analytic algorithms and the need to continue to increase the sensitivity and specificity of these algorithms.

For future development of predictive algorithms, Zimmet et al. highlight the importance of using data from multiple NICUs. They extracted heart rate, pulse rate derived from pulse oximetry, and oxygen saturation obtained from continuous monitoring of 1168 very low birth weight infants in 3 institutionally unrelated NICUs from birth through day 42 (35,238 infant-days of data). Their data suggest that vital sign patterns can be impacted not only by onset of illness but also by NICU-specific care practices, monitor management and hardware, and socioeconomic/race/ethnicity characteristics of infants. Using vital sign data from a single NICU risks development of predictive algorithms that reflect NICUspecific characteristics and are not generalizable across NICUs. The ability to detect inter-NICU differences from vital sign data also suggests their usefulness for generation of multi-component, predictive algorithms that can identify, standardize, and monitor best practices across NICUs.

The vital sign signatures observed by Zimmet et al. identified both consistent patterns across NICUs and inter-NICU differences associated with differences in care delivery, monitor management and hardware, and infant characteristics. They found consistent vital sign patterns across all 3 NICUs in heart rate and oxygen saturation metrics, in heart rate increases of $\sim 10$ beats/minute (from 150 to 160 beats/min) over the first 2 weeks of life, and in lack of clinically significant differences in vital signs between sexes after correction for birth weight, gestational age, and institution. Their heart rate data confirm and extend prior observations in fullterm infants ${ }^{12}$ and in preterm infants from a single NICU. ${ }^{13}$ Consistency of heart rate metrics across the 3 NICUs in the first 2 weeks of life suggests that caffeine use is similar although no patient-specific data are available. The consistency of mean oxygen saturation over time ( $94 \%)$ in all 3 NICUs reflects the use of standardized NICU guideline targets based on large clinical trials. ${ }^{7-10}$ However, in contrast to the consistency of these vital sign patterns, the frequencies of bradycardia (heart rate $<100$ beats/min for $\geq 4 \mathrm{~s}$ ) and desaturation (oxygen saturation $<80 \%$ for $\geq 10 \mathrm{~s}$ ) events differed in the first 2 weeks of life among the 3 nurseries: infants at one NICU had up to twice as many bradycardia events per day during the first 2 weeks of life, while infants at another NICU had about half as many desaturation events. The inter-NICU differences in bradycardia events were not detectable after 3 weeks of age, while the differences in daily number of desaturation events between sites increased from birth to 6 weeks of age. The authors speculate that the lower number of bradycardia events at one of the NICUs is associated with differences in ventilator care practices: the NICU with lower use of mechanical ventilation and greater use of nasal continuous positive airway pressure had a greater number of bradycardia events. Although this speculation is supported by differences in the average number of days on mechanical ventilation per infant among the 3 NICUs ( 10 days vs. 35 days vs. 33 days in 2017-2018), confirmation in this study was not possible due to lack of availability of patient-specific, daily respiratory support data. The authors point to differences in alarm alert management and in socioeconomic/race/ethnicity characteristics of infants among the NICUs to explain the differences in desaturation events.

For future development of vital sign-based, standardized predictive algorithms that can be used across NICUs, this report highlights the importance of using data from NICUs with differing infant demographics, clinical practices, and monitor management and characteristics. In addition, vital sign data described by Zimmet et al. highlight their usefulness for identification of interNICU differences in nursing- and physician-based care practice, infant race/ethnicity, outcome, and infant socioeconomic characteristics. Linkage of vital sign data with other accessible sources of electronic data can advance discovery of standardized best practices across NICUs, reduce preventable harm, and improve outcomes. ${ }^{14,15}$ For example, the California Perinatal Quality Care Collaborative (CPQCC) and the Vermont Oxford Network have provided standardized quality metrics that have measurably and consistently improved outcomes through inter-NICU comparisons and best practice identification. ${ }^{16,17}$ However, the data in the manuscript by Zimmet et al. provide an example of the importance of including race/ethnicity and socioeconomic data linked to social determinants of health for future development of predictive algorithms. While the CPQCC publishes a health equity dashboard and has observed racial and/or ethnic variation in quality of care, ${ }^{18,19}$ other predictive analytic algorithms have not systematically included race/ethnicity or social determinants of health metrics. ${ }^{20}$ For example, the impact of omission of race/ ethnicity data on bronchopulmonary dysplasia (BPD) risk estimation in the National Institute of Child Health and Human Development (NICHD) BPD Outcome Estimator ${ }^{21,22}$ was recently described. ${ }^{23,24}$ Specifically, Whitehead et al. illustrate a paradox of the NICHD BPD Risk Estimator that resulted from omission of recognized disparities in preterm birth risk and mortality between

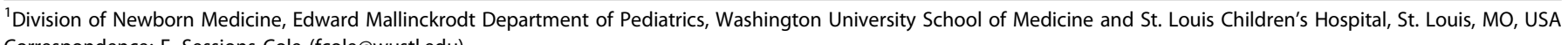
Correspondence: F. Sessions Cole (fcole@wustl.edu)

Received: 28 February 2021 Accepted: 3 March 2021

Published online: 13 April 2021 
African- and European-descent preterm infants. This omission leads to a sufficiently high mortality projection for African-descent infants that the threshold risk of moderate-to-severe BPD is never attained despite high severity of illness. The authors point out that, without adjustment for race-based disparities in mortality risk, prediction algorithms run the risk of erroneous risk estimation due to the competing risk of increased mortality. Inclusion of race/ ethnicity and social determinants of health data in predictive algorithms will be important in identifying NICU strategies to reduce racial disparities in multiple infant health outcomes. ${ }^{22,25,26}$

Nursing practice data are also critical for inter-NICU comparisons of clinical care delivery processes ${ }^{27}$ but are not routinely included in large clinical data sets used for predictive algorithm development. The diversity of nursing practice data collection platforms among NICUs and the vendor-specific, proprietary differences between platforms have reduced electronic access to standardized nursing practice data collection. ${ }^{28}$ Future predictive analytic algorithms should take advantage of open-source data dictionaries for data collection that include intentional integration of nursing practice data. ${ }^{28}$ Zimmet et al. highlight the importance of using vital sign data from multiple NICUs for future development of predictive risk algorithms with improved sensitivity and specificity. Their work also suggests the importance of integrating vital sign-based analytics with other NICU-specific characteristics of physician- and nurse-based care delivery, monitor management and hardware, infant socioeconomic status, unit culture, and race/ ethnicity to accelerate discovery and implementation of standardized best practices across NICUs that will improve infant outcomes through regional, national, and international collaboration. ${ }^{14,15,29-31}$

\section{ADDITIONAL INFORMATION}

Competing interests: The author declares no competing interests.

Publisher's note Springer Nature remains neutral with regard to jurisdictional claims in published maps and institutional affiliations.

\section{REFERENCES}

1. Kumar, N., Akangire, G., Sullivan, B., Fairchild, K. \& Sampath, V. Continuous vital sign analysis for predicting and preventing neonatal diseases in the twenty-first century: big data to the forefront. Pediatr. Res. 87, 210-220 (2020).

2. Moorman, J. R. et al. Mortality reduction by heart rate characteristic monitoring in very low birth weight neonates: a randomized trial. J. Pediatr. 159, 900-6 e1 (2011).

3. Stone, M. L. et al. Abnormal heart rate characteristics before clinical diagnosis of necrotizing enterocolitis. J. Perinatol. 33, 847-850 (2013).

4. Griffin, M. P., Lake, D. E., O'Shea, T. M. \& Moorman, J. R. Heart rate characteristics and clinical signs in neonatal sepsis. Pediatr. Res. 61, 222-227 (2007).

5. Fairchild, K. D. et al. Vital signs and their cross-correlation in sepsis and NEC: a study of 1,065 very-low-birth-weight infants in two NICUs. Pediatr. Res. 81, 315-321 (2017)

6. Saria, S., Rajani, A. K., Gould, J., Koller, D. \& Penn, A. A. Integration of early physiological responses predicts later illness severity in preterm infants. Sci. Transl. Med. 2, 48ra65 (2010).

7. Saugstad, O. D. \& Aune, D. Optimal oxygenation of extremely low birth weight infants: a meta-analysis and systematic review of the oxygen saturation target studies. Neonatology 105, 55-63 (2014).
8. Askie, L. M. et al. Association between oxygen saturation targeting and death or disability in extremely preterm infants in the neonatal oxygenation prospective meta-analysis collaboration. JAMA 319, 2190-2201 (2018).

9. Poets, C. F. et al. Association between intermittent hypoxemia or bradycardia and late death or disability in extremely preterm infants. JAMA 314, 595-603 (2015).

10. Di Fiore, J. M. et al. Patterns of oxygenation, mortality, and growth status in the surfactant positive pressure and oxygen trial cohort. J. Pediatr. 186, 49-56 e1 (2017).

11. Sullivan, B. A. et al. Early pulse oximetry data improves prediction of death and adverse outcomes in a two-center cohort of very low birth weight infants. Am. J. Perinatol. 35, 1331-1338 (2018).

12. Fleming, $\mathrm{S}$. et al. Normal ranges of heart rate and respiratory rate in children from birth to 18 years of age: a systematic review of observational studies. Lancet $\mathbf{3 7 7}$, 1011-1018 (2011).

13. Alonzo, C. J. et al. Heart rate ranges in premature neonates using high resolution physiologic data. J. Perinatol. 38, 1242-1245 (2018).

14. Kaempf, J., Morris, M., Steffen, E., Wang, L. \& Dunn, M. Continued improvement in morbidity reduction in extremely premature infants. Arch. Dis. Child. Fetal Neonatal Ed. https://doi.org/10.1136/archdischild-2020-319961 (2020).

15. Lee, H. C. et al. Comparison of collaborative versus single-site quality improvement to reduce NICU length of stay. Pediatrics 142, e20171395 (2018).

16. Lee, H. C., Liu, J., Profit, J., Hintz, S. R. \& Gould, J. B. Survival without major morbidity among very low birth weight infants in California. Pediatrics 146, e20193865 (2020).

17. Edwards, E. M., Ehret, D. E. Y., Soll, R. F. \& Horbar, J. D. Vermont Oxford Network: a worldwide learning community. Transl. Pediatr. 8, 182-192 (2019).

18. Gould, J. B. Building the first statewide quality improvement collaborative, the CPQCC: a historic perspective. Children 7, 177 (2020).

19. Profit, J. et al. Racial/ethnic disparity in NICU quality of care delivery. Pediatrics 140, e20170918 (2017).

20. Rysavy, M. A. et al. Assessment of an updated neonatal research network extremely preterm birth outcome model in the Vermont Oxford Network. JAMA Pediatr. 174, e196294 (2020).

21. Laughon, M. M. et al. Prediction of bronchopulmonary dysplasia by postnatal age in extremely premature infants. Am. J. Respir. Crit. Care Med. 183, 1715-1722 (2011).

22. Davis, J. M. \& Pursley, D. M., Pediatric Policy C. Preventing long-term respiratory morbidity in preterm neonates: is there a path forward? Pediatr. Res. 87, 9-10 (2020).

23. Whitehead, H. V. et al. The challenge of risk stratification of infants born preterm in the setting of competing and disparate healthcare outcomes. J. Pediatr. 223, 194-196 (2020).

24. Vesoulis, Z. A., McPherson, C. C. \& Whitehead, H. V. Racial disparities in calculated risk for bronchopulmonary dysplasia: a dataset. Data Brief. 30, 105674 (2020).

25. Litt, J. S., Fraiman, Y. S. \& Pursley, D. M. Health equity and the social determinants: putting newborn health in context. Pediatrics 145, e20200817 (2020).

26. Beck, A. F. et al. The color of health: how racism, segregation, and inequality affect the health and well-being of preterm infants and their families. Pediatr. Res. 87, 227-234 (2020).

27. Lake, E. T., Staiger, D., Edwards, E. M., Smith, J. G. \& Rogowski, J. A. Nursing care disparities in neonatal intensive care units. Health Serv. Res. 53, 3007-3026 (2018).

28. Singh, $\mathrm{H}$. et al. Development of data dictionary for neonatal intensive care unit: advancement towards a better critical care unit. JAMIA Open 3, 21-30 (2020).

29. Lui, K. et al. Inter-center variability in neonatal outcomes of preterm infants: a longitudinal evaluation of 298 neonatal units in 11 countries. Semin. Fetal Neonatal Med. https://doi.org/10.1016/j.siny.2021.101196 (2021).

30. Pursley, D. M. \& McCormick, M. C. Bending the arc for the extremely low gestational age newborn. Pediatr. Res. 83, 751-753 (2018).

31. Profit, J. et al. The correlation between neonatal intensive care unit safety culture and quality of care. J. Patient Saf. 16, e310-e316 (2020). 